\title{
DEEP LEARNING FOR CURVE AND DROWSY DETECTION IN HILL TERRAIN
}

\author{
Dr A. Akilandeswari \\ Associate Professor, ECE \\ St Joseph's Institute of \\ Technology Chennai, India
}

\author{
Deepika R S \\ B.E, ECE \\ St Joseph's Institute of \\ Technology Chennai, India
}

\author{
Kannika M \\ B.E, ECE \\ St Joseph's Institute of \\ Technology Chennai, India
}

\begin{abstract}
We are developing an embedded product that helps in prevention of accidents. Our product has concentrated on two aspects to prevent accidents. First, Drowsiness of drivers. Second, Narrow curves on roads of hills. In order to prevent accidents because of these two aspects our product has been proposed. For preventing accidents because of drowsiness we have implemented image processing based on Eye Aspect Ratio (EAR) [1], it monitors driver and alert them. For preventing at curves we have implemented camera that traps the radius of curve and the vehicle coming opposite. This system also comprises MEMS sensor, GPS, GSM to send message in case of emergency.
\end{abstract}

Keywords: Image processing, perspective transform, MEMS sensor, EAR (Eye Aspect Ratio).

\section{INTRODUCTION}

In recent years, the development of automobile industries has resulted in a major deployment of automobiles in both developing and developed countries. Despite the fact that quality of automobiles nowadays has been improved in a higher rate, there are still some low cost and low speed models that are operated in developing countries. The main reason behind this is that those expensive models are attracted by the top earners alone, as the mid-range people can only afford low cost automobiles. In order to rescue Automobiles from accidents by drowsiness of drivers or hill terrains, this research aims to develop an enhanced real-time system. The algorithm supports processing of various images of drivers as well as the processing of road images.

The remaining of this paper is organized as follows: Section II details about the block diagram of system. Section III presents the proposed algorithm. Section IV discusses results and presents the analysis. Finally, Section V presents conclusions and some suggestions for future works.

\section{BLOCK DIAGRAM}

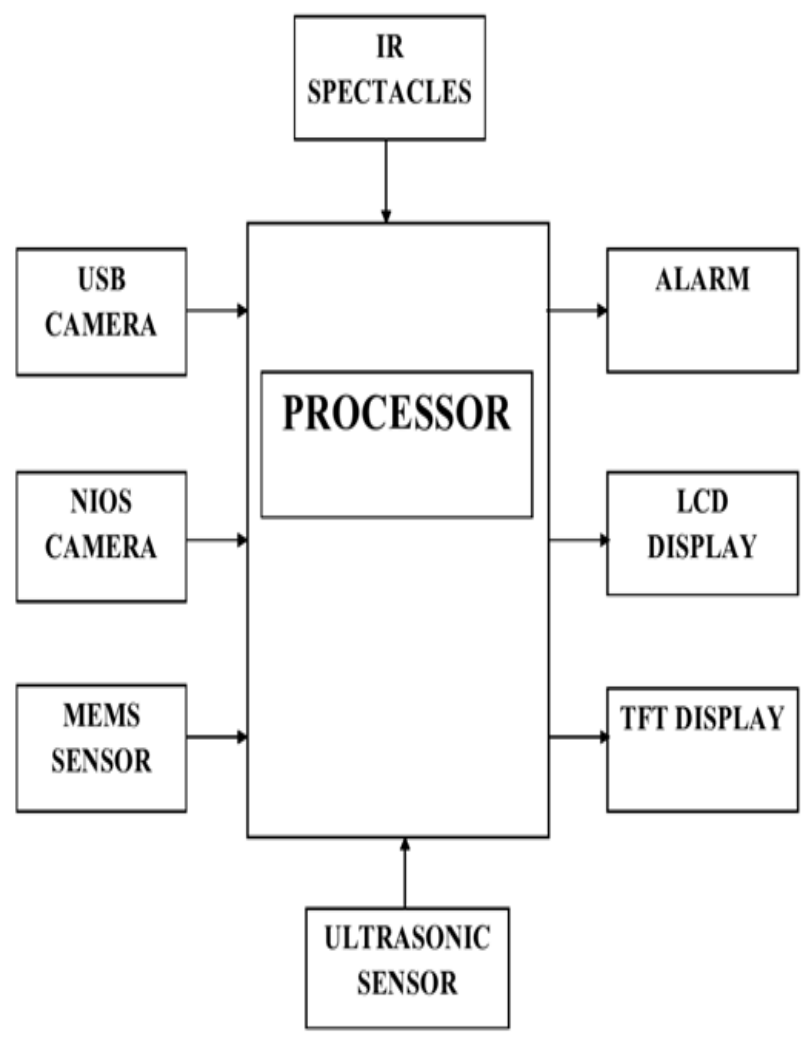

Fig. 1. Block diagram of the system

The simple block diagram of our project has been described in this section. Processor is main processing unit; processor will be with maximum clock speed, because it tends to 
process images within fraction of seconds. IR spectacles and USB camera is for capturing driver face, for defining whether driver is drowsy or not.

Then the NIOS camera for capturing lane, which will get as the entire detail of road, that would help the diver by intimation in TFT display. Alarm, wake up the driver. LCD display will intimate others about driver condition.

\section{METHODOLOGY}

1. Lane detection:

First part of the project starts with the detection of lane. The process goes on by; Fix the capture image into chessboard for clarification [2]. Mostly the chessboard of 9X6 is usually used for clearance. Then convert the image to undistorted 2D image for processing. Binary threshold is the key concept for detecting the path of the vehicle. The goal is to identify pixels that are likely to be part. That is similar shades will resemble in unique color, and different shades shows variation. Help to identify the different objects present on lane. Absolute horizontal and vertical sobel [9] used to find the magnitude and normal horizontal and vertical sobel used to find the gradient. All these used to find binary threshold image by converting RGB to BINARY. This binary transformation is a process of converting the gray image into binary with some threshold values. If the obtained value is our required threshold, then perspective transformation is carried out. The perspective transformation ${ }^{[3]}$ plays role for lane detection. Perspective means "when human eyes see near things they look bigger as compare to those who are far away". Perspective is the process of converting 3-D into a 2-D surface image such that height, width, depth and position can be related to each other in other to determine the lane and curvature effectively.

This consists of transformation of 5 frames ${ }^{[7]}$. Object coordinates (3D) -> World coordinates (3D) $\rightarrow>$ Camera coordinates (3D) $\rightarrow$ Image plane coordinates (2D) -> Pixel coordinates (2D, int).

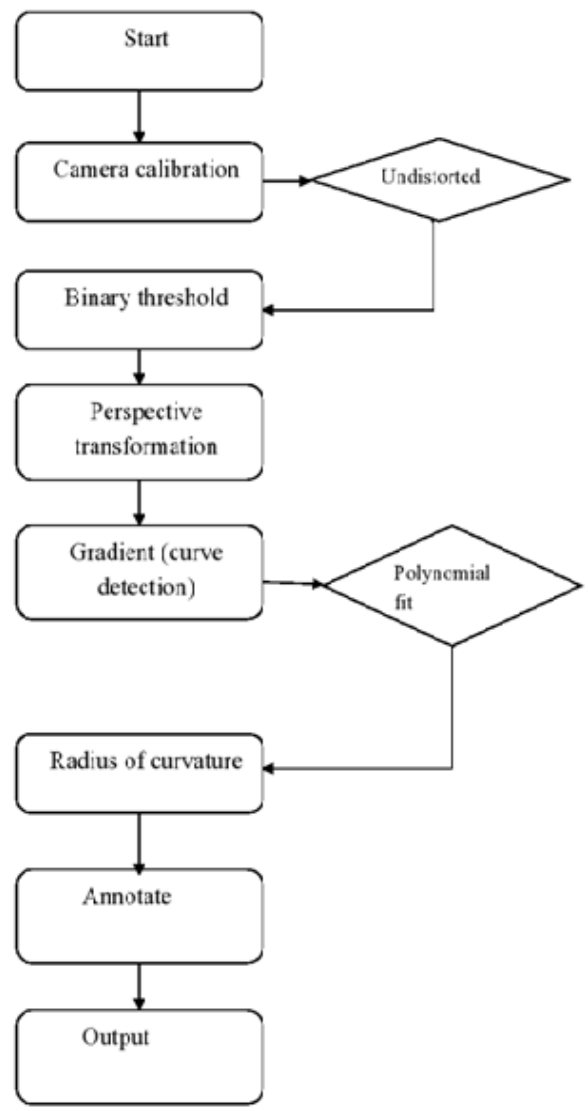

Fig. 2. Flow chart of lane detection

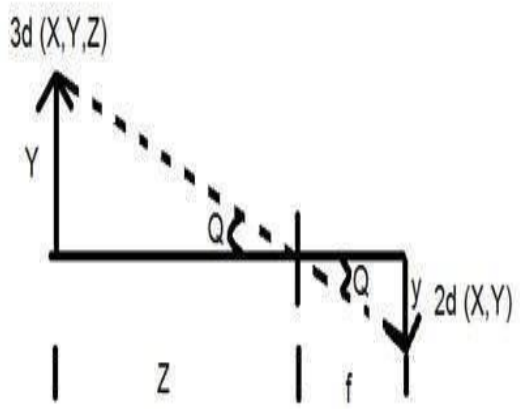

Fig. 3. Perspective analysis of image

$$
\begin{aligned}
& Y=3 d \text { object } \\
& y=2 d \text { Image } \\
& f=\text { focal length of the camera } \\
& Z=\text { distance between object and the camera }
\end{aligned}
$$

Here perspective means fit to polynomial. That 


\section{International Journal of Engineering Applied Sciences and Technology, 2020 \\ Vol. 5, Issue 8, ISSN No. 2455-2143, Pages 147-152 \\ Published Online December 2020 in IJEAST (http://www.ijeast.com)}

is the lane path is detected, cropped and it is fixed with suitable polynomial so that it can be used for further processing and calibration purpose. With respect to histogram ${ }^{[4]}$ and curve present the image is portioned into 9 slices. Based on slices comparison the prefect curve of road is taken out.

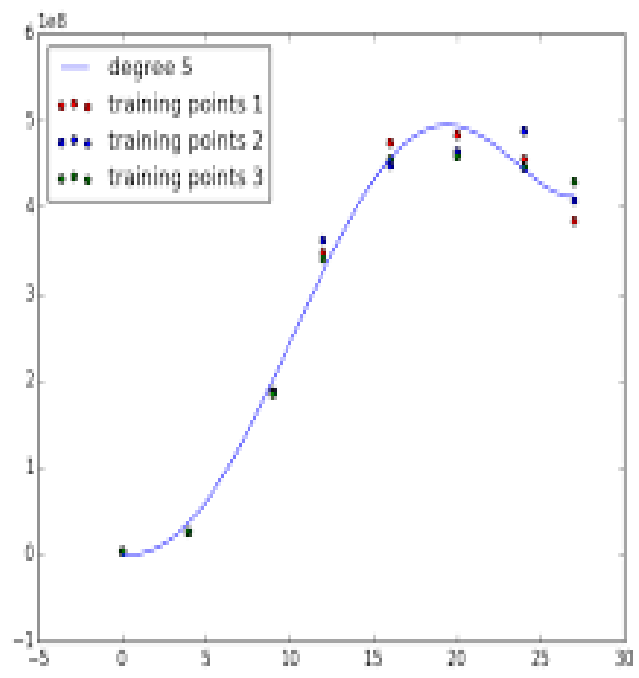

Fig. 4. Determination of radius of curvature

Radius of the curve is another vital part that plays a role in output of the display. It is calculated by different measures, which are based on assumptions as follow: Pixies to meters Vertical $30 \mathrm{~m} / 720$ pixels \& horizontal $3.7 \mathrm{~m} / 700$ pixels

For finding the radius of the curvature ${ }^{[5]}$ we go with the application of differential:

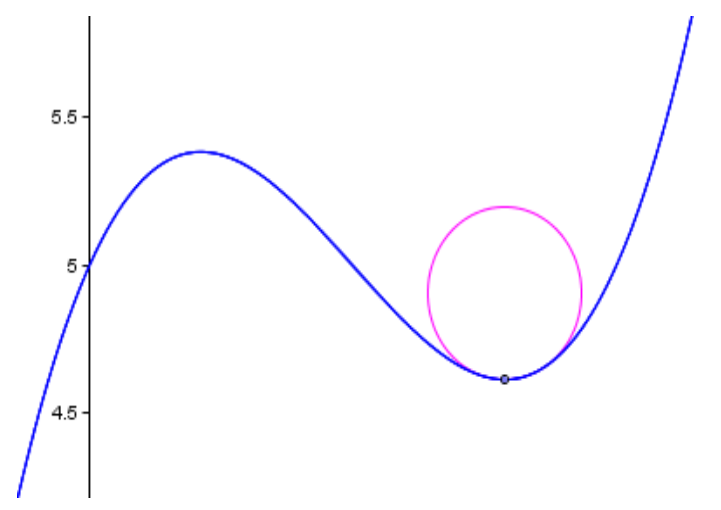

Fig. 5. Differential analysis ${ }^{[1]}$

$$
Y=f(x)=\left\{1+(d y / d x)^{2}\right\}^{3 / 2}
$$

3. Drowsy detection:

Second have the concept of eye detection. Eye detection starts with the processing IR spec. whenever eye blink of the driver crossed the threshold, it automatically starts the camera, we use the Logitech camera.

The image is captured and the frame of $15 \mathrm{sec}$ is taken together for a set. The frames are interconnected and convoluted to form the shape prediction. This shape prediction is relying on dlib, which is trained before it. The shape prediction is important so that the face of the person is detected which help to identify the eyes. It follows the skim of ROI ${ }^{[15]}$ using general image filtering. After identifying the ROI ${ }^{[15]}$, either based on probability or average distance, eye is detected.

Then the method goes with counting the blink of the eye. It is calculated using the algorithm EAR (Eye Aspect Ratio) the formula is mentioned below:

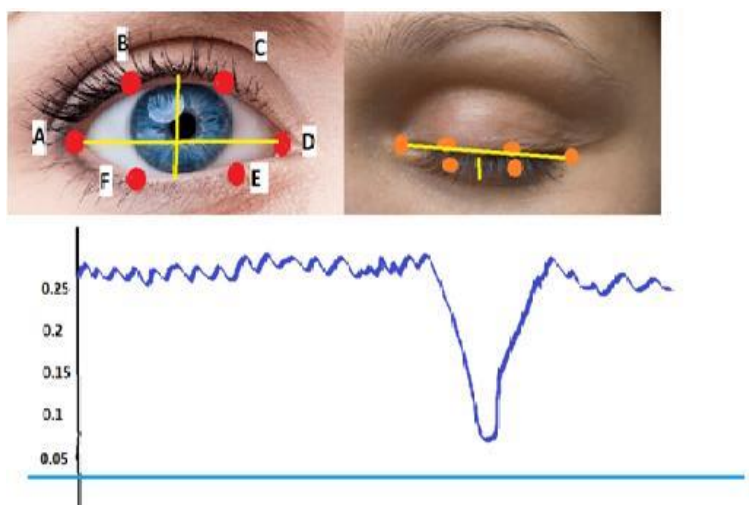

Fig. 6. EAR detection of eye
A- $\mathrm{D}=$ horizontal distance of eye
$\mathrm{B}-\mathrm{F}=$ vertical distance of eye
$C-E=$ vertical distance of eye
$\mathrm{EAR}=\frac{(\mathrm{B}-\mathrm{F})+(\mathrm{E}-\mathrm{C})}{2(\mathrm{~A}-\mathrm{D})}$

If eye is open then the EAR will be constant, if the eye is closed then the EAR value will be suddenly set to zero. With respect to this the blink count is taken. Roughly EAR is set to 0.13 because it is suitable for various humans 
as a mean value.

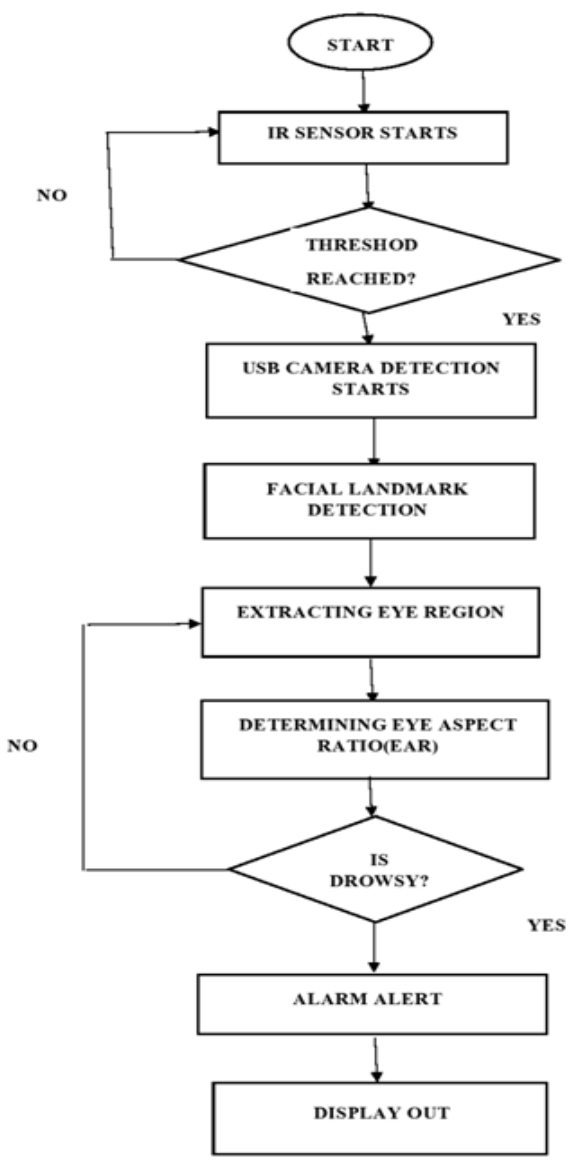

Fig. 7. Flow chart for drowsy detection

Frames and EAR is set within certain threshold so that the correct identification of drowsiness of driver is found. If the threshold is attained then the alarm will start, and it can be turned off only by the person. Then the digital display outside the vehicle will display about the condition of the driver.

Final processes of our project relays on MEMS sensor ${ }^{[6]}$, which helped to know about the roll off of the vehicle, below image will give simple working

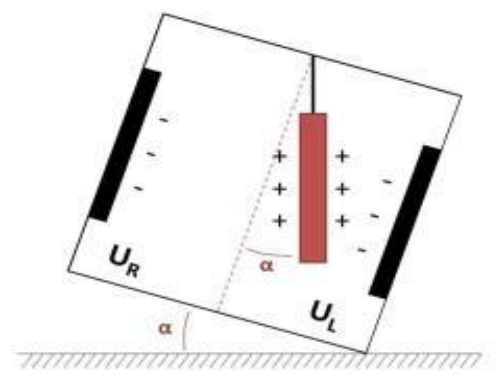

Fig. 8. MEMS detection for wheel rollover

As the vehicle tire roll on the road, the capacitor inside the MEMS sensor will change according and notify the driver about the tire. It helps in prevention from the accident.

These methods are incorporated into a single embedded system. This will help the driver to prevent accident. Display outside the vehicle help others to know about the exact condition of the driver.

\section{RESULT}

In this method, the drowsiness and lane is scanned continuously. Lane detection is the primary process, and the drowsy occurred as interrupt with higher priority. The lane of the road in which the driver is driving will also be detected. The curvature of the bends will be displayed to the drivers such that the driver can take up the correct turn and the curvature can be known early such that speed of the vehicle can be controlled accordingly by the drivers. Especially in hill terrain this output will be more helpful for the divers to avoid accidents. Under drowsy, the webcam or mobile camera kept in front of the drivers will be continuously determining the frames using EAR algorithm. If the frame fails to reach the threshold value, then an alarm is provided to wake up the driver. The above methodologies are considered to be a high speed process. Advantage of this product is that MEMS sensor is provided near the wheel which has the ability to detect the roll off of the wheel. In case of any irrelevant actions of the wheel, these MEMS provide a warning message to the controller. This will help the driver to take necessary actions before any accident occurs. 
International Journal of Engineering Applied Sciences and Technology, 2020

Vol. 5, Issue 8, ISSN No. 2455-2143, Pages 147-152

Published Online December 2020 in IJEAST (http://www.ijeast.com)

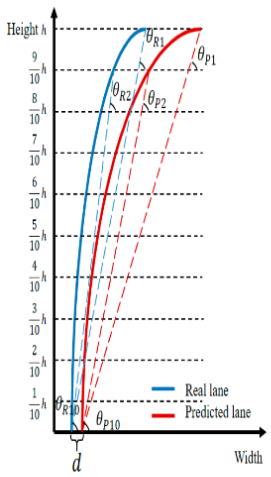

(a)

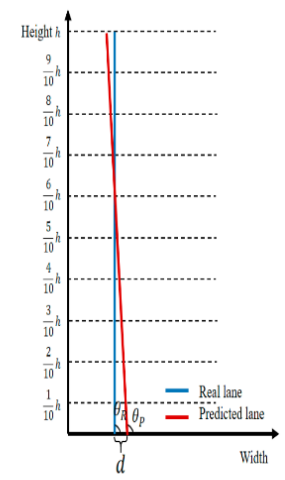

(b)
Fig. 9. Estimated graph for Lane Detection ${ }^{[8]}$

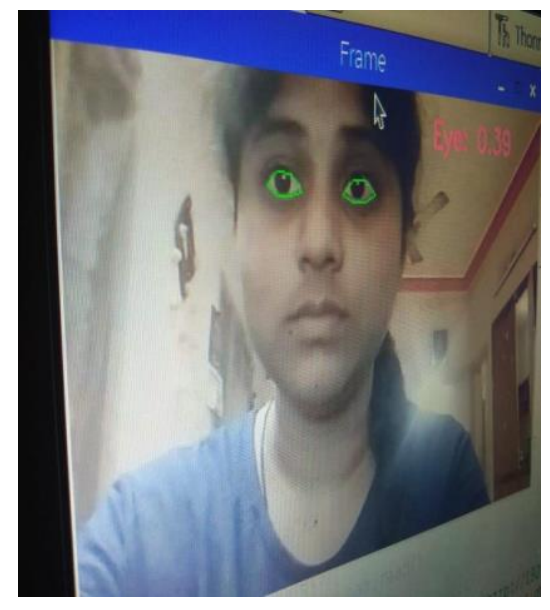

Fig.10. Eye frame calculation

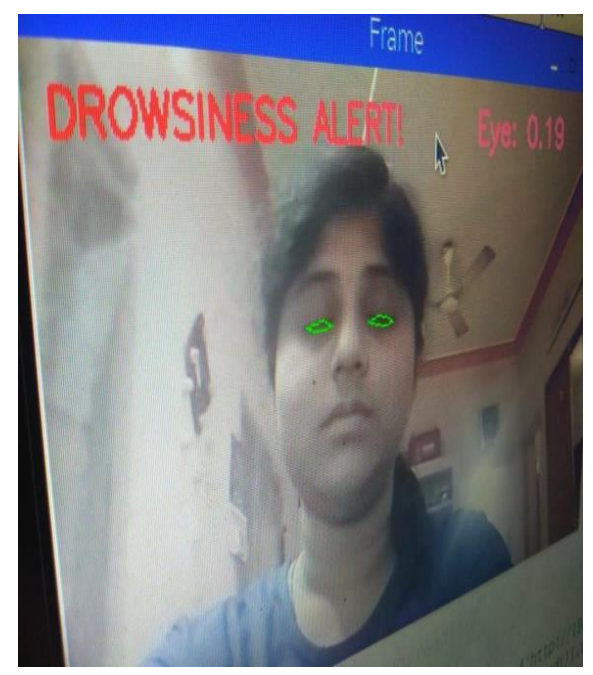

Fig.11. Drowsy detection using EAR ratio

\section{CONCLUSION}

The fact is that most of the accidents are mainly due to the drowsiness of the drivers. The drivers are not able to control the vehicle when they fall asleep. In highways, mostly the drivers would drive at a very high speed due to which handling is tough and controlling the speed in such a condition is difficult. Another most accident causing reason is, in hill terrains in due to unaware about the curves.

In this product we use Deep learning for curve detection and image processing for drowsiness detection as the key principle.

These methodologies are high speed processes, that provides high responses and detect ability. The main components of this product are frames for drowsy and perspective transform for curve. Therefore, we propose a high speed product which uses advanced technologies to safeguard the drivers.

\section{REFERENCE}

1)https://www.pyimagesearch.com/2017/05/08/dr o wsiness-detection-opencv/

2)https://opencv-python-

tutroals.readthedocs.io/en/latest/py_tutorials/py_c al ib3d/py_calibration/py_calibration.html

3)https://www.tutorialspoint.com/dip/perspective _t ransformation.htm

4)IJCSNS International Journal of Computer Science and Network Security, VOL.12 No.8, August 2012 Manuscript received August 5, 2012

Manuscript revised August 20, 2012 Histogram Based Circle Detection Saleh Basalamah Umm Al- Qura University, Makkah, Saudi Arabia

5)https://www.intmath.com/applicationsdifferentiation/8-radius-curvature.php

6)A Comprehensive Study on Technologies of Tyre Monitoring Systems and Possible Energy Solutions Ali E. Kubba1, * and Kyle Jiang 2Sensors 2014,

$14,10306-10345$

7)https://www.tutorialspoint.com/dip/perspective _t ransformation.html 
9)https://medium.com/datadriveninvestor/underst an ding-edge-detection-sobel-operator2aada303b900

10) Lane Detection of Curving Road for Structural High-way with Straight-curve Model on Vision. IEEE Transactions on Vehicular Technology, 1-1 Wang, H. feng, Wang, Y. fei, Zhao, X., Wang, G. ping, Huang, H., \& Zhang, J. (2019).

11)https://www.intmath.com/applicationsdifferentiation/8-radius-curvature.php

12) Lane and vehicle lane departure detection. 2017 2nd IEEE International Conference on Recent Trends in Electronics, Information \& Communication Technology (RTEICT). Rao, N., \& Tasgaonkar P P (2017).

13) Real-time lane-vehicle detection and tracking system. 2016 Chinese Control and Decision Conference(CCDC). Guan, H., Xingang, W., Wenqi, W., Han, Z., \& Yuanyuan, W. (2016).

14) Vehicle lane detection and following based on vision system and laser scanner. 2017 International Conference on Applied System Innovation (ICASI). Huang, S.-J., \& Tsai, C.-C. (2017).

15)http://en.wikipedia.org/wiki/Region_of_intere st 